\title{
HHT based analysis on seismic response recordings for a base-isolated building
}

\author{
Fei Wang*, Xiandong Kang, Ting Yan, and Ying Liu \\ Beijing Earthquake Agency, Beijing 100080, China
}

\begin{abstract}
Hilbert-Huang transform (HHT) is proposed to process the seismic response recordings in an 8-story frame-shear wall base-isolated building. Empirical Mode Decomposition (EMD) method is first applied to identify the time variant characteristics and the data series can be decomposed into several components. Hilbert transform is well-behaved in identifying the frequency components. The first 5 intrinsic mode functions (IMFs) are decomposed with their different frequencies. The analytical function is reconstructed and compared with the original signal. They are extremely consistent in amplitude and phase. Based on the IMFs obtained, frequencies of the original signal are inferred at $5 \mathrm{~Hz}$ and $1.6 \mathrm{~Hz}$. The higher frequency is regarded as the vibration excited by surface waves. 1.6 $\mathrm{Hz}$ is suggested as the dominant frequency of the building. Analysis indicates that HHT is accurate in extracting the dynamic characteristics of structural systems.
\end{abstract}

\section{Introduction}

Structural vibration signals are commonly analyzed to extract the dynamic characteristic parameters including natural frequency, damping ratio and mode shapes [1]. Structural health states are identified based on the changes of structural dynamic characteristics. By comparing the characteristics of the undamaged and original structures, the damages are detected. Various methods including Fast Fourier transform (FFT) and wavelet transform have been proposed for natural characteristics identification in structural health monitoring and diagnosis. Fast Fourier transform is an analysis method in frequency domain and suitable for analyzing stable time series in linear process. However, it has obvious deficiency in nonlinear process due to low resolution and external noise in fitting original time series. Wavelet transform realizes analysis in time domain by transforming the signals with scalable and translatable wavelets [2,3]. Wavelet analysis is essentially a Fast Fourier transform with adjustable windows and signals in time windows are required to be stationary. Therefore, it has the similar limitations with Fast Fourier transform $[4,5]$.

The method of Hilbert-Huang transform (HHT) has been developed to analyze signals in the frequency-time domain. It is consisted of Empirical Mode Decomposition (EMD) and Hilbert transform. Thereinto, EMD is the critical part for data analysis. HHT method has already been proved more precise than the wavelet analysis[6,7]. Furthermore, HHT method is applicable to process non-stationary time series. In this study, HHT is introduced

* Corresponding author: wangfei@bjseis.gov.cn 
to analyze the structural seismic response recordings in an 8-story base-isolated building and the inherent dynamic characteristics are identified.

\section{Basic principle of HHT}

The method of EMD is crucial to HHT and it was recently proposed to decompose data series $x(t)$ into intrinsic mode functions (IMFs). An IMF has two basic requirements. The number of extreme points and the number of zero crossings for the entire data must be equal or must not be more than one if they differ. The mean of the upper and lower envelopes is computed to be zero at any extreme point. The IMF components can be determined by repeated application of sifting process, an iterative procedure. Han and Li repeated the process for HHT and applied to analyse the data in shake table test [8].

For data series $\mathrm{x}(\mathrm{t})$, by introducing $\mathrm{h}_{1}$ it can be sifted firstly as

$$
\mathrm{h}_{1}=\mathrm{x}(\mathrm{t})-\mathrm{m}_{1} \text {. }
$$

Here $m_{1}$ stands for Instantaneous mean of the upper and lower envelopes. Usually $h_{1}$ does not meet the basic requirements. Therefore, the sifting process can be repeated $\mathrm{k}$ times and $\mathrm{h}_{1 \mathrm{k}}$ is obtained meeting the IMF requirements. The first IMF component $\mathrm{C}_{1}$ is computed out with the component of highest frequency,

$$
\mathrm{r}_{1}=\mathrm{x}(\mathrm{t})-\mathrm{C}_{1} \text {. }
$$

Analysis is carried out with $r_{1}$ instead of $x(t)$ and the second IMF component is obtained. The signal can be finally decomposed into $\mathrm{n}$ IMFs and the mean trend of the signal or a constant as $r_{n}(t)$, indicated in

$$
x(t)=\sum_{j=1}^{n} C_{j}(t)+r_{n}(t) .
$$

$\tilde{\mathrm{x}}(\mathrm{t})$ is the Hilbert transform of $\mathrm{x}(\mathrm{t})$,

$$
\tilde{x}(t)=H T[x(t)]=\frac{1}{\pi} P \int_{-\infty}^{+\infty} \frac{x(\tau)}{t-\tau} d \tau .
$$

Here $\mathrm{P}$ stands for Cauchy principal value. Analytical signal of $\mathrm{x}(\mathrm{t}), \mathrm{Y}(\mathrm{t})$ is calculated as,

$$
Y(t)=x(t)+i \tilde{x}(t)=A(t) e^{i \theta(t)}
$$

$A(t)$ is denominated as the instantaneous amplitude, $\theta(t)$ instantaneous phase angle and $\omega(t)$ instantaneous frequency. They can be computed out as

and

$$
\begin{gathered}
A(t)=\left[x^{2}(t)+\tilde{x}^{2}(t)\right]^{1 / 2}, \\
\theta(t)=\arctan \left(\frac{\tilde{x}(t)}{x(t)}\right),
\end{gathered}
$$

Now $Y(t)$ can be transformed into

$$
\omega(\mathrm{t})=\mathrm{d} \theta(\mathrm{t}) / \mathrm{dt}
$$

$$
Y(t)=\sum_{j=1}^{n} A_{j}(t) e^{i \int \omega_{j}(t) d t}=\sum_{j=1}^{n} A_{j}\left(t, \omega_{j}\right) e^{i \int \omega_{j}(t) d t} .
$$

$A_{j}\left(t, \omega_{j}\right)=$ amplitude of the $j$ th IMF at time $t$ with frequency $\omega_{j}$. Thus, the amplitude of the frequency-time decomposition of $\mathrm{x}(\mathrm{t})$, denoted by $\mathrm{A}(\mathrm{t}, \omega ; \mathrm{x})$ is given by

$$
A(t, \omega ; x)=\sum_{j=1}^{n} A_{j}\left(t, \omega_{j}\right) \text {. }
$$

$A(t, \omega ; x)$ is referred to as the Hilbert-Huang spectrum of $x(t)$. The marginal spectrum, denoted by $h(\omega, x)$, is obtained as

$$
h(\omega, x)=\int_{0}^{\infty} A(t, \omega ; x) d t,
$$

which is the decomposition of $\mathrm{x}(\mathrm{t})$ in the frequency domain. Consequently, each IMF can be calculated using the Hilbert transform to obtain the first $n$ different frequency components at any time. HHT is applied in processing the seismic response recordings in a base-isolated building and the dominant frequencies are inferred. 


\section{Building profile}

The base-isolated building is frame-shear wall structure with 8 stories and 2 basements. The total height is 33.0 meters and the plan view is shown in figure 1. An intense seismic response array was instrumented in the building with 33 sensors on basement 2, 1st floor, 3rd floor, 6th floor and the roof. Five 3-channel sensors are installed on the geometrical center and six 3-channel sensors are on the two ends of the building. A 3-channel seismometer was deployed in a 100-meter borehole. Sensor layout is demonstrated in figure 2.

\section{Seismic response recordings}

After the array was instrumented, several earthquakes were recorded including the earthquake at the border of Baodi District, Tianjin and Yutian County, Hebei in China. The earthquake occurred in June 18th, 2012 with magnitude 4.0. The epicenter distance was approximately 180 kilometers and the amplitudes of the recordings are small. The seismic response recordings of the building were corrected and shown in figure 3 . The peak acceleration is $0.20 \mathrm{~cm} / \mathrm{s}^{2}$ in North-South direction and $0.21 \mathrm{~cm} / \mathrm{s}^{2}$ in East-West direction.

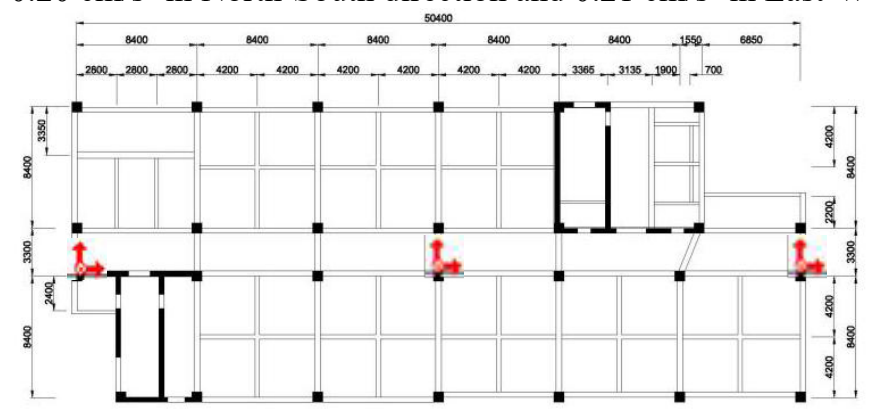

Fig. 1. structure plan view. Red arrows represent the sensor and the monitoring direction.

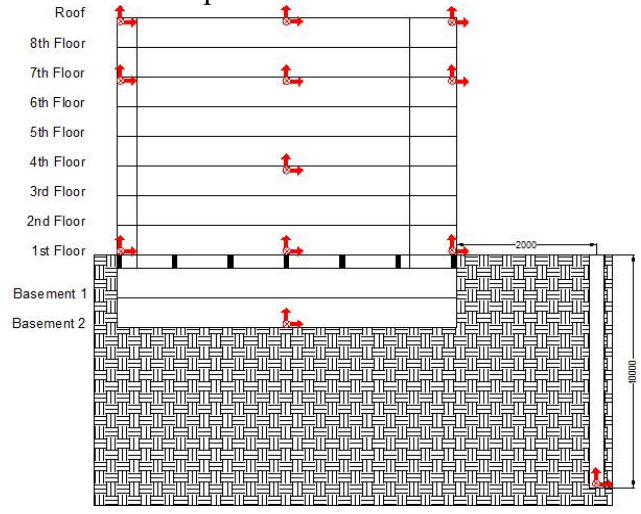

Fig. 2. layout of 36 sensors in the building. Red arrows represent the sensor and the monitoring direction. 


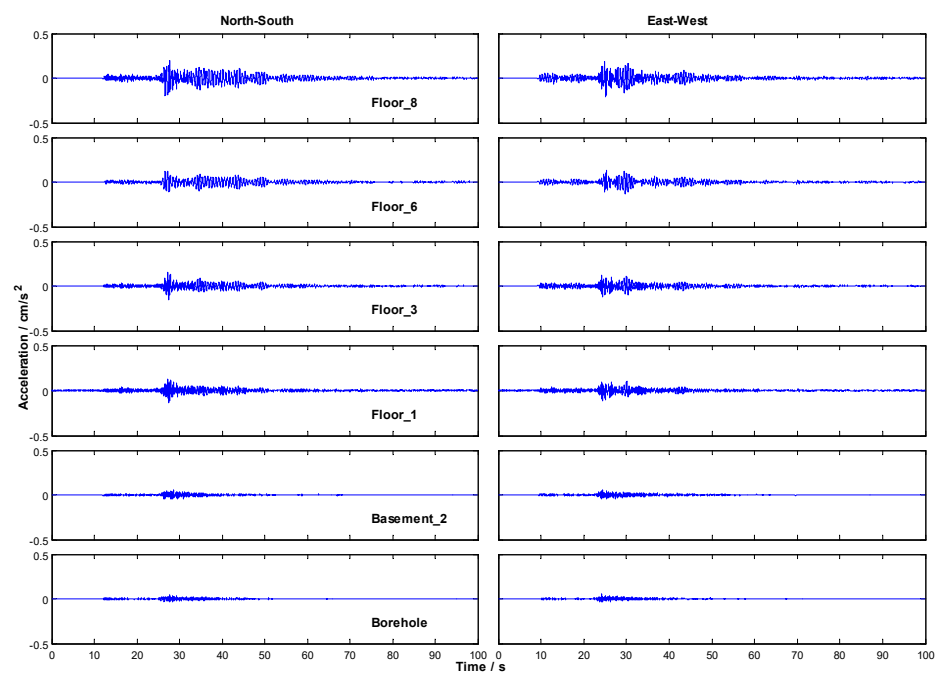

Fig. 3. time-histories of seismic response recordings in North-South and East-West directions.

\section{HHT analysis}
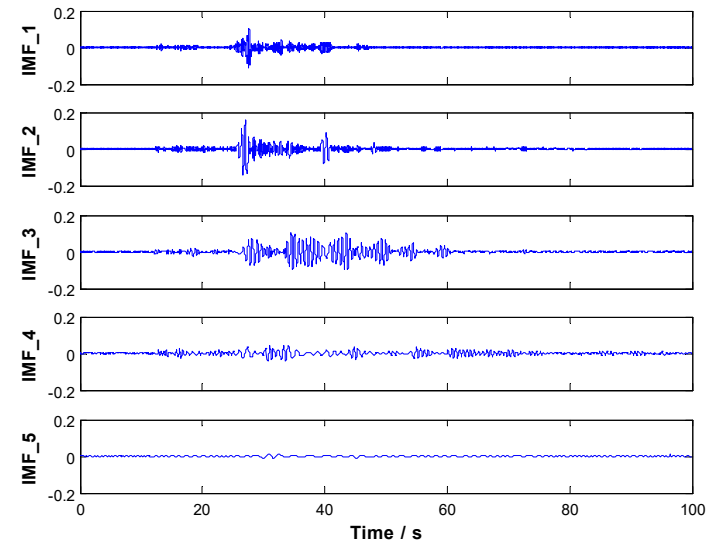

Fig. 4. IMFs obtained in EMD analysis defined from IMF_1 to IMF_ 5 with the unit as $\mathrm{cm} / \mathrm{s}^{2}$.
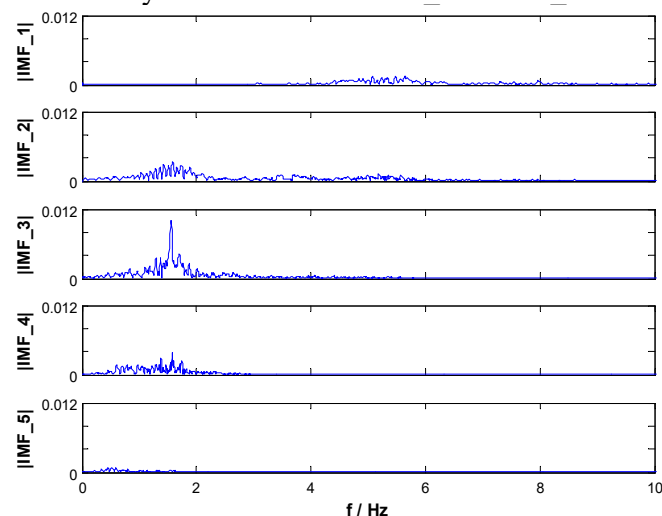

Fig. 5. Hilbert transform of the IMFs and the frequency contents for each IMF with the unit as amplification factor. 
The time-history in North-South direction on the $8^{\text {th }}$ floor is selected for HHT analysis. EMD is applied to the recording and the first 5 IMFs are decomposed. They are nominated from IMF_1 to IMF_5. Figure4 demonstrates the IMFs and IMF_5 becomes almost vanished. The first 4 empirical mode components of the recording are dominated at a specific frequency throughout the time history. After EMD analysis, Hilbert transform is used to each IMF and the frequency components are obtained for all IMFs, as indicated in figure 5. Herein, IMF_1 contains higher component and the frequency decreases as the number of IMF increases. Frequency component in IMF_5 is greatly weakened.

To verify the effectiveness in component decomposition, the first 5 IMFs are summated and the analytical function is reconstructed. It is compared with the original signal. The plot in figure 6 suggests the differences between the analytical function and the original signal. The main vibration from $25 \mathrm{~s}$ to $50 \mathrm{~s}$ are extracted and demonstrated. Tiny differences indicate that 5 cyclic analyses for IMFs are adequate to obtain the majority frequency components. Therefore, all the frequencies in different IMF are added together and the frequency component of the analytical function is determined. The frequency contents of the first 3 IMFs represent the frequency components in the recorded seismic response. As shown in figure 7 , the dominant frequency of the building under this earthquake is implied as $1.6 \mathrm{~Hz}$. The dominant frequency was analysed with the value of $1.62 \mathrm{~Hz}$ using other analytical method. The difference is $1.3 \%$. They are considered to be consistent. There is another frequency between $4 \mathrm{~Hz}$ and $6 \mathrm{~Hz}$. It is regarded as the vibration excited by the surface wave. As shown in figure 5, it is the frequency of the IMF_1 and it is visible, but very weak.

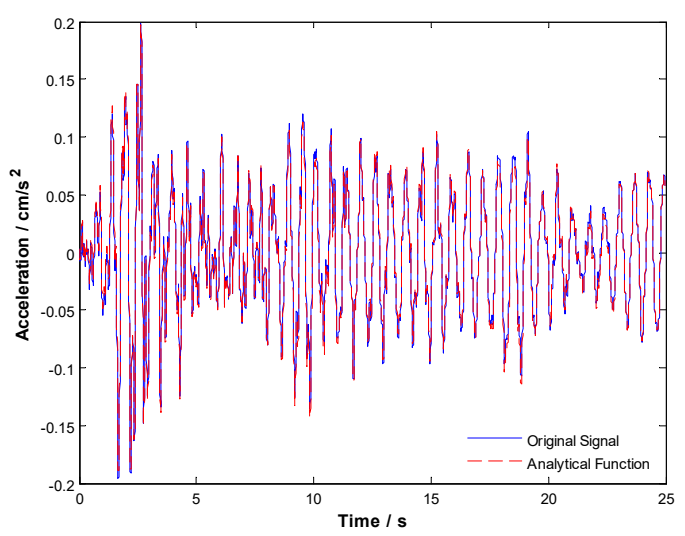

Fig. 6. Comparing between the seismic response recording on the $8^{\text {th }}$ floor and its analytical function.

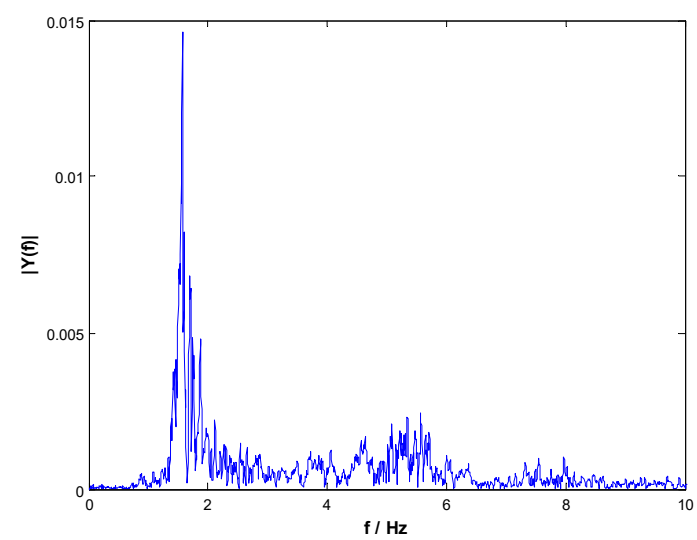

Fig. 7. The frequency content of the analytical function and the dominant frequency is $1.6 \mathrm{~Hz}$. 


\section{Conclusion}

Hilbert transform can be used to identify the time variant characteristics of the structural system. The data series have to be decomposed into its components to admit a well-behaved Hilbert transform. EMD method is first implemented for component decomposition. The EMD method and Hilbert transform construct HHT, which is developed to analyse signals in the frequency-time domain. HHT is introduced to process the seismic response recordings in an 8-story frame-shear wall base-isolated building. EMD method is applied to decompose the frequency content of the signal on the roof and 5 IMFs are inferred from higher frequencies to small ones. The components with their frequencies are indicated after Hilbert transform. The origin signal and its analytical function are compared and no obvious differences are detected. The HHT analysis can achieve satisfactory accuracy in reconstructing the origin signal and inferring the frequency contents.

\section{Acknowledgement}

We would like to thank the anonymous reviewers for thorough reviews, which helped to improve the technical quality and presentation of this article. Special thanks are extended to Beijing Earthquake Bureau for providing the waveform data. We also thank Erol Kalkan for sharing his computer codes, which we modified significantly for this study. This study is financially supported by Project of Science for Earthquake Resilience, China Earthquake Administration, Granted No. XH17001.

\section{References}

1. Huan S L, Mcinnis B C, and Denman E D 1983 Analysis of the random decrement method Int. J. Syst. Sci. 14 (4) pp 417-423

2. Abdulkareem M, BakharyN, Vafaei M 2015 Application of Wavelet Transform to Damage Detection in Plates using Response-only Measurements In 16th Asia Pacific Vibration Conference pp 24-26

3. Vafaei M, Alih S C, Rahman A B A, Adnan A B 2015 A wavelet-based technique for damage quantification via mode shape decomposition Structure and Infrastructure Engineering 11(7) pp 869-883

4. J. N. Yang, Y. Lei, S. Lin, and N. Huang 2004 Hilbert-Huang Based Approach for Structural Damage Detection JOURNAL OF ENGINEERING MECHANICS $130 \mathrm{pp}$ 85-95

5. Ridong Du , Yongbo Yuan and Miao Chen 2012 Empirical Mode Decomposition Application for Structural Seismic Responses Applied Mechanics and Materials and Materials256-259, pp 2096-2101

6. Huang N E et al. 1998 The empirical mode decomposition and hilbert spectrum for nonlinear and nonstationary time series analysis Proc. R. Soc. London Ser A 454 pp 903-995

7. Huang N E, Shen Z and Long S R 1999 A new view of nonlinear water waves The Hilbert spectrum Annu Rev Fluid Mech. 31 pp 417-457

8. Jianping Han and Dawen Li 2010 Modal parameter identification based Hilbert-Huang Transform and natural excitation technique ENGINEERING MECHANICS 27 (8) pp 54-59 